\title{
From fibroblast cells to cardiomyocytes: direct lineage reprogramming
}

Lei Yang*

\begin{abstract}
Recent advances in stem cell biology have established the feasibility of reprogramming human and murine fibroblast cells into induced pluripotent stem cells. Three master regulators have been demonstrated to be sufficient in the management of cell status of 'pluripotent' versus 'differentiated'. The same strategy has been used to directly convert one somatic cell type into another cell type, such as the converting of exocrine pancreas cells into cells closely resembling beta cells and the reprogramming of fibroblast cells into functional neuron cells. Srivastava's group reported the first direct reprogramming of mouse fibroblast cells into mesoderm lineage cells (cardiomyocytes) with the enforced expression of three cardiac transcriptional factors: Gata4, Mef2c, and Tbx5. The induced cardiomyocytes exhibit a global gene expression profile and basic electrophysiological characteristics similar to those of postnatal cardiomyocytes. This study made significant advances in cardiovascular and stem cell fields and has important implications in understanding heart developmental biology as well as in potential therapies of human cardiovascular diseases.
\end{abstract}

Recent advances in stem cell biology have established the feasibility of reprogramming human and murine fibroblast cells into induced pluripotent stem (iPS) cells [1-3]. The ectopic expression of four transcription factors (Oct4, Sox2, c-Myc, and Klf4) in fibroblasts was shown to be efficacious in the conversion of fibroblast cells into embryonic stem (ES) cell-like status. Generation of iPS cells ushers in a new era in reprogramming differentiated somatic cells, including fibroblasts, neural cells, liver cells, stomach cells, and blood cells, into ES cell-like stem

*Correspondence: lyang@pitt.edu

Department of Developmental Biology, University of Pittsburgh, 530 45th Street, 8117 Rangos Research Center, Pittsburgh, PA 15201, USA cells [4]. With the progress of iPS technology, the concept of 'master regulators', defined as a group of major reprogramming factors playing a critical role in the management of cell status of 'pluripotent' versus 'differentiated', has been demonstrated. The group of master regulators for iPS cell generation is found to be effective with only three genes [5], which is far fewer than the hundreds or thousands of genes that were presumed to be involved in the determination of cell fate or status.

Successful reprogramming of fibroblast cells into iPS cells raised the possibility of directly converting one somatic cell type into other cell types. By overexpressing Ngn3, Pdx1, and Mafa, Zhou and colleagues [6] reported the conversion of exocrine pancreas cells into cells closely resembling beta cells and having the function of secreting insulin. In 2010, successful reprogramming of fibroblast cells into functional neuron cells was reported with the enforced expression of Ascl1, Brn2, and Myt1l [7]. Recently, Srivastava's group [8] used the same strategy and reported another breakthrough in direct reprogramming of mouse fibroblast cells into beating cardiomyocytelike cells: the transduction of a set of three cardiac master factors important in early heart development (Gata4, Mef2c, and Tbx5). This is the first paper to reveal the possibility of directly committing fibroblast cells into heart muscle cells that exhibit electrophysiological characteristics similar to those of adult cardiomyocytes. By comparing the transcription profile of mouse cardiomyocytes with that of cardiac fibroblasts, Srivastava and colleagues [8] selected 14 key cardiac specific transcriptional factors as the starting pool. In addition, the authors isolated the Thy $1^{+} / \alpha M H C-G F P^{-}$fibroblast cells from the neonatal mouse heart. The 14 factors were introduced into Thy $1^{+} / \alpha M H C-G F P^{-}$cardiac fibroblast cells with retro-virus, and $\alpha \mathrm{MHC}-\mathrm{GFP}^{+}$cells were shown after 1 week of transduction, indicating the cardiac potential of reprogrammed fibroblast cells. Of the 14 factors, Gata4, Mef2c, and Tbx5 were identified as being sufficient for cardiomyocyte reprogramming and having the

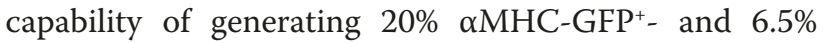
cTNT $^{+}$-induced cardiomyocytes (iCMs). The iCMs show a clear sarcomeric organization and a global gene expression pattern similar to those of postnatal mouse 
cardiomyocytes. The iCMs also gain an epigenetic status similar to that of cardiomyocytes, indicating that the fibroblasts were epigenetically converted to cardiomyocytelike cells.

Considering the possibility that iCMs could be derived from the subtle heart progenitor cells existing within the Thy $1^{+} / \alpha M H C-G F P^{-}$cardiac fibroblast cells, Srivastava's group induced iCMs from mouse tail dermal fibroblast

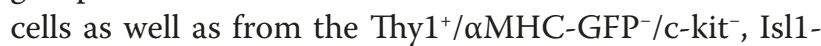
YFP/Thy1 $1^{+}$, and Mesp1-YFP-/Thy $1^{+}$fibroblast cells. c-kit, Isl1, and Mesap1 have been used as markers of the cardiac progenitor cells and the early mesodermal cells before cardiac commitment. Direct reprogramming of iCMs from the above fibroblast cells strongly supported the view that iCMs originated from differentiated fibroblast cells, not from a subpopulation of heart progenitors. Most interestingly, the iCMs exhibit electrophysiological characteristics resembling those of adult ventricular cardiomyocytes. As human ES cell- and iPS cell-derived cardiomyocytes show phenotypes of fetal cardiomyocytes $[9,10]$, the adult cardiomyocyte-like characteristics of iCMs could provide more clues in understanding how to induce ES cell- and iPS cell-derived cardiomyocytes into a mature status.

This paper paved the way for the generation of a large number of cardiomyocyte-like cells and has important implications in understanding heart developmental biology as well as in the potential therapies of human cardiovascular diseases. For example, global knockouts of key cardiac transcriptional factors, such as Isl1 [11], exhibit embryonic lethality. Whether iCMs could be induced from fibroblast cells of the global knockout embryos and whether the efficiency of iCMs could be affected by the knockout genes are very interesting questions in the field of developmental biology. This paper [8] also reported that cardiac fibroblast cells can give rise to a much higher frequency of iCMs than the tail fibroblast cells can. Determining whether the 'tissue memory' affects the reprogramming and elucidating what the mechanism is would also be interesting questions. The future generation of iCMs from humans, especially from patients with genetically caused cardiovascular diseases, would provide a unique system in which to understand those inherited diseases. However, there are still some issues to be addressed in the direct reprogramming of iCMs. The first is how closely the $\mathrm{iCMs}$ can resemble the electrophysiological characteristics of adult cardiomyocytes. Activation of the $\mathrm{Ca}^{2+}$ channel in iCMs was reported. How about the activities of $\mathrm{K}^{+}$and $\mathrm{Na}^{+}$channels in iCMs? Second, this paper reported the induction of approximately $25 \%$ of $\alpha \mathrm{MHC}_{\mathrm{GFP}}{ }^{+}$cells, and only $5 \%$ to $6 \%$ of $\mathrm{cTNT}^{+}$ cardiomyocytes after the direct reprogramming. Future studies need to improve the reprogramming efficiency. The third is whether ectopic expression of the three reprogramming factors in the adult mouse heart will lead to the abnormal formation of cardiac myocytes in vivo and whether heart stem cells could be activated through a similar mechanism. The fourth is whether iCMs from mouse heart disease models, such as models of hypertrophic cardiomyopathy and arrhythmias, will exhibit similar in vivo disease phenotypes.

\section{Abbreviations}

ES, embryonic stem; iCM, induced cardiomyocyte; iPS, induced pluripotent stem.

\section{Competing interests}

The authors declare that they have no competing interests.

Published: 14 January 2011

References

1. Takahashi K, Yamanaka S: Induction of pluripotent stem cells from mouse embryonic and adult fibroblast cultures by defined factors. Cell 2006, 126:663-676.

2. Takahashi K, Tanabe K, Ohnuki M, Narita M, Ichisaka T, Tomoda K, Yamanaka S: Induction of pluripotent stem cells from adult human fibroblasts by defined factors. Cell 2007, 131:861-872.

3. Yu J, Vodyanik A, Smuga-Otto K, Antosiewicz-Bourget J, Frane JL, Tian S, Nie J, Jonsdottir GA, Ruotti V, Stewart R, Slukvin II, Thomson JA: Induced pluripotent stem cell lines derived from human somatic cells. Science 2007, 318:917-920.

4. Müller LU, Daley GQ, Williams DA: Upping the ante: recent advances in direct reprogramming. Mol Ther 2009, 17:947-953.

5. Nakagawa M, Koyanagi M, Tanabe K, Takahashi K, Ichisaka T, Aoi T, Okita K, Mochiduki Y, Takizawa N, Yamanaka S: Generation of induced pluripotent stem cells without Myc from mouse and human fibroblasts. Nat Biotechnol 2008, 26:101-106.

6. Zhou Q, Brown J, Kanarek A, Rajagopal J, Melton DA: In vivo reprogramming of adult pancreatic exocrine cells to beta-cells. Nature 2008, 455:627-632.

7. Vierbuchen T, Ostermeier A, Pang ZP, Kokubu Y, Südhof TC, Wernig M: Direct conversion of fibroblasts to functional neurons by defined factors. Nature 2010, 463:1035-1041.

8. leda M, Fu JD, Delgado-Olguin P, Vedantham V, Hayashi Y, Bruneau BG, Srivastava D: Direct reprogramming of fibroblasts into functional cardiomyocytes by defined factors. Cell 2010, 142:375-386.

9. Yang L, Soonpaa M, Edler E, Roepke T, Kattman S, Kennedy M, Henckaerts E, Bonham K, Abbott G, Lindon M, Field L, Keller G: Human cardiovascular progenitors develop from a KDR+ ES cell-derived population. Nature 2008 453:524-528.

10. Zhang J, Wilson GF, Soerens AG, Koonce CH, Yu J, Palecek SP, Thomson JA, Kamp TJ: Functional cardiomyocytes derived from human induced pluripotent stem cells. Circ Res 2009, 104:30-41.

11. Cai CL, Liang X, Shi Y, Chu PH, Pfaff SL, Chen J, Evans S: Isl 1 identifies a cardiac progenitor population that proliferates prior to differentiation and contributes a majority of cells to the heart. Dev Cell 2003, 5:877-889.

doi:10.1186/scrt42

Cite this article as: Yang L: From fibroblast cells to cardiomyocytes: direct lineage reprogramming. Stem Cell Research \& Therapy 2011, 2:1. 